\title{
La chronicité des cancers permet-elle une vie enviable ?
}

\author{
Does the Chronicity of Cancer Allow for an Enviable Life?
}

\author{
M.-F. Bacqué \\ (C) Lavoisier SAS 2019
}

\section{Le passage au temps long}

Les cancers changent progressivement de statut avec les améliorations des traitements et de la prise en soins. Une majorité prétend maintenant au qualificatif enviable de « maladie chronique ». Pourtant, les auteurs de ce numéro spécial sur la chronicité observent que ce terme peut « camoufler » une mortalité qui ne porte plus son nom ou désigner une nouvelle temporalité thérapeutique. «Garder son cancer toute sa vie », « être, toute sa vie, surveillé médicalement », «ne plus quitter son traitement » sont les expressions relevées ici, quand santé et maladie jouent un subtil duo, à moins qu'une certaine hypocrisie ne règne afin d'éviter les mots qui fâchent.

Nous serions donc passés de maladie mortelle à maladie chronique, d'espoir de guérison à espoir de soulagement. Les malades du cancer, après avoir perçu le « vent du boulet » lors de son annonce, comme le disaient joliment les Anglo-Saxons au début de la description du psychotraumatisme, souhaitent toutefois se débarrasser définitivement du cancer et de son univers. Leur offrir une maladie " chronique $»$ ne les convainc pas. Notre voyage en oncologie nous montre en effet que le changement de vocabulaire ne laisse personne dupe, en particulier lorsqu'il s'agit de supporter les effets secondaires des traitements et les conséquences irréversibles physiques et psychiques des cancers.

\section{Acculturation à la maladie chronique}

Psychologues, psychiatres et oncologues cherchent comment parler du cancer «persistant » ou « toujours présent mais endormi » aussi bien aux adultes qu'aux enfants. Ils constatent que c'est bien de la temporalité dont il faut parler.

M.-F. Bacqué $(\bowtie)$

SuLiSoM, faculté de psychologie,

12, rue Goethe, F-67000 Strasbourg, France

e-mail : mfbacque@club-internet.fr
$\mathrm{Si}$, le cancer est contenu et contrôlé, il pourrait cependant se manifester à nouveau. Pour éviter une rechute, il semble nécessaire de se retrouver régulièrement afin d'observer le nouvel équilibre du patient avec ses risques d'évolution vers la maladie.

Plus que jamais la maladie humaine doit faire l'objet d'acculturation. Comme pour tout changement de pays ou de langue, les femmes et les hommes atteints de cancer ont besoin de saisir les niveaux différents de cette affection, le niveau génique, cellulaire, d'organe, de fonction, puis de vie et de mort. Ne demandons pas évidemment à chacun de devenir biologiste ou médecin, mais trouvons les mots pour faciliter la compréhension du processus carcinogène. Au-delà de cette description, trouvons les personnes, thérapeutes ou compagnons de route susceptibles d'échanger et surtout d'écouter les interrogations des malades. Enfin, face à la réalité, proposons les moyens de supporter le temps qui passe : entretiens, groupes de partage, divertissement, création.

\section{La mort, issue taboue à aborder comme un questionnement éthique}

Ainsi, alors que la possibilité de rendre vivable la chronicité d'un état non désiré est explorée quotidiennement à l'hôpital et surtout à la maison, l'évolution de la société française rappelle qu'il est possible de mettre fin à sa souffrance en demandant le recours à une sédation profonde et continue jusqu 'à la mort. À nous de conserver le recours au questionnement éthique en équipe et de résister au passage à l'action individuelle, délétère en toute circonstance.

C'est dans ce but que la Société Française de PsychoOncologie a rédigé ses propositions pour l'analyse psychologique des demandes de sédation continue jusqu'au decès. Notre méthode est toujours axée sur la discussion avec le patient, discussion inclusive, éclairée et articulée sur les autres échanges facultatifs mais à proposer : ouverture à la famille et à l'équipe soignante élargie (comprenant le médecin de famille). Les débats et enjeux exceptionnels qui 
entourent, depuis plus de dix ans, l'état végétatif de Vincent Lambert ne doivent pas oblitérer la possibilité de parler sereinement de la fin de la vie. Les premières paroles à ce sujet, encore fragiles aujourd'hui, devraient encadrer une des questions majeures de nos vies : celle de nos directives anti- cipées. Ici aussi, notre approche est celle du temps long. Le questionnement fait partie de notre psychodynamique : conscient de notre mort, nous butons devant son irruption. Elle frappe à la porte, on s'écrie : encore un peu de temps ! Non, la vie n'est justement pas chronique, seule la maladie l'est... 